\title{
Lane Departure Warning System for Vehicle Active Safety Perception Platform
}

\author{
Jia HE \\ No.218. Chnenglin Road. Dongli District. Tianjin, PR. China \\ E-mail: hejia@catarc.ac.cn
}

Keywords: Vehicle Active Safety Perception Platform; Lane departure warning; Image processing

\begin{abstract}
The vehicle active safety perception platform is a effective way to prevent vehicle accident. Lane Departure Warning System (LDWS) is one of the most important aspects of this system. In this paper, a set of image processing method which can give results fast and accurately in the non-ideal conditions is proposed. And this is composed of a CMOS camera, an image acquisition and processing module, and an interface module. Several algorithms are tested as the edge detection method according to the experiment comparison, and other several algorithms are tested as the efficient way to detect the lane. At last, experimental facilities installed in the test vehicle validates the performance of this vehicle active safety perception platform.
\end{abstract}

\section{Introduction}

SVRD (Single Vehicle Road Departure) accidents accounts for a high proportion of all accidents on the highways. According to statistics from AASHTO (American Association of State Highway and Transportation Officials), SVRD accidents took around 20 percent of traffic accidents and 40 percent of fatalities in total in the United States in 2006.

According to the research report from Daimler-Benz, the reasons of traffic accidents can be classified into five columns: Road Departure (account for 19\% of all traffic accidents), Lane Change and Merge (account for 4\%), Intersection (account for 29\%), Rear End (account for 26\%), and other reasons account for about $22 \%$. Study also shows that over $80 \%$ accidents had relationship with the driver's unintended or dozing, and if 0.5 second warning can be provided, $60 \%$ accidents can be avoided. If 1 second warning can be provided, 90\% accident can be avoided [1].

Lane Departure Warning System (LDWS) is one of the most important aspects of Intelligent Vehicle (IV) technology, which is an active safety system to prevent unintended lane departure that can lead single vehicle roadway departure, lane change/merge, and rollover crashes. LDWS uses a forward looking monocular camera to acquire lanes information for determining the position of the vehicle relative to the lanes [2][3]. If a lane departure is happening and the vehicle's turn signal is not in use, LDSW gives the driver a warning, when a vehicle is running above a certain speed threshold.

\section{Lane Departure Warning System Structure}

The LDWS is a part of the Vehicle Active Safety Perception Platform, and this part is composed of a CMOS camera, an image acquisition and processing module, and an interface module. The camera that captures front view images is installed between the front windshield and the rear-view mirror inside the experimental vehicle. The image acquisition and processing module is a digital media processor based on DaVinci ${ }^{\mathrm{TM}}$ technology. Road lane images can be acquired by the CMOS camera and transmitted to a digital signal processor after the decoding process from NTSC analog format to digital format. The DSP core will identify and estimate with the internal algorithm to give the vehicle position and direction to the lane. The system predicts the time interval before the vehicle departs the current lane, namely Time to Lane Cross (TLC) [4].When the system finds SVRD happened, it will give a warning as the results shown on the LCD displayer and the warning sounds made by the alarm buzzer. Fig.1 shows the structure of the Vehicle Active Safety Perception Platform. 


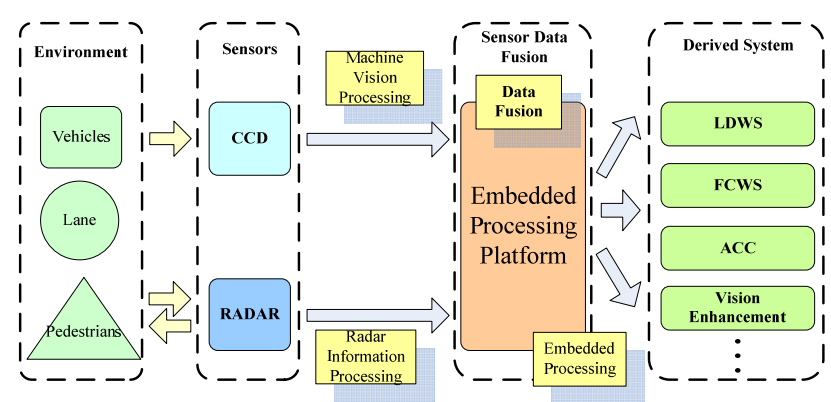

Figure 1. Structure of the Vehicle Active Safety Perception Platform

The camera that captures front view images is installed between the front windshield and the rearview mirror inside the experimental vehicle. In order to get the correspondence between the image and the real road, the relational model between the two-dimensional image information and threedimensional world information must be established [5].

Suppose the road in front of the vehicle is flat and the camera is installed in a fixed location. Two Euclidean spaces are defined: the three-dimensional world coordinate system $\mathrm{W}$ and the twodimensional image coordinate system I.

$$
\begin{aligned}
& W=\{(x, y, z)\} \in E^{3} \\
& I=\{(u, v)\} \in E^{2}
\end{aligned}
$$

Once the lane markings were recognized in the image plane, the road modeling for lane markings can be calculated by using coordinates conversion equations (3) (5)

$$
\begin{aligned}
& x(u, v)=h \times \cot \left[(\theta-\alpha)+u \frac{2 \alpha}{R_{v}-1}\right] \times \sin \left[(\gamma-\alpha)+v \frac{2 \alpha}{R_{u}-1}\right]+l \\
& y(u, v)=h \times \cot \left[(\theta-\alpha)+u \frac{2 \alpha}{R_{v}-1}\right] \times \cos \left[(\gamma-\alpha)+v \frac{2 \alpha}{R_{u}-1}\right]+d \\
& z(u, v)=0
\end{aligned}
$$

\section{Image Processing}

After obtain the image information in front of the vehicle, firstly extract lane mark features by image processing, and then fit the lane from these features. In practice, light, dodging and a variety of complex noise make it very difficult to detect lanes fast and accurately, so one of the LDWS key technologies is to establish a set of image processing method which still can give results fast and accurately in the conditions of image information obtained non-ideal [6].

\section{A. Edge Detection}

The edge is the junction region between one property region from another in the image, which is the most basic image characteristic. Essentially, the image edge reflect non-continuity of local image characteristics (grayscale mutation, color mutation, etc.). It marks the end of one region and beginning of another. Edges are where the area properties change suddenly with most uncertainty in the image. Edge detecting can reduce the amount of data and filter out useless information, while preserving the important structural properties in the image. Therefore, edge detecting plays a key role in image processing, analyzing and recognizing.

There are many edge detection algorithms; , and the class of differential operator edge detection method are the most basic edge detection methods. This kind of method is more concise, with less calculated amount, and very efficient. Therefore, the differential operator edge detection method is a very useful tool which has become one of the most active subjects in machine vision research field and has occupied a very important role in the engineering application. There are several common edge detection operators: Roberts operator, Sobel operator, Prewitt operator, Kirsch operator, GaussLaplace operator, and Canny operator.

Sobel operator is a first order differential operator, which can be used for fast convolution function simply and efficiently, so it has become an extremely popular edge detection operator. Sobel operator 
has the advantages of simple, processing fast, edge smooth, continuous, positioning accurate and less error. So Sobel operator has practical value in real-time applications such as lane detection in Intelligent Vehicle systems.

Sobel edge detection operator is to take weighted average firstly, then make differential, and then seek gradient. The Sobel edge detection operator comes from two convolution kernels, and each pixel in the image make convolution integral with these two kernels. One of them has the most impact on vertical edges, and the other one has the most impact on horizontal edges. The center of the operator corresponds to the center pixel, and then makes convolution integral with it. The maximum of two convolution kernels is taken as the output of the point. The computing result is the edge of the image. In edge detection, Sobel operator weights the impact of pixel position. The weighted average border width is larger than 2 pixels.

The Canny edge detection algorithm [7] is known to many as the optimal edge detector. Canny's intentions were to enhance the many edge detectors already out at the time he started his work. He was very successful in achieving his goal. Canny follows principles to improve the current edge detection methods. The first principle is a lower error rate. It is important that edges occurring in images should not be left out and that there should be no response to non-edges. The second principle is that the edge point should be localized well. In other words, the distance between the edge pixels and the actual edge should be as small as possible. The third principle is that each single edge has only one response.

Based on these criteria, the Canny detector first smoothes the image to eliminate and noise. It then finds the image gradient to highlight regions with high spatial derivatives. The algorithm then tracks along these regions and suppresses any pixel that is not at the maximum (non-maximum suppression). The gradient array is now further reduced by hysteresis. Hysteresis is used to track along the remaining pixels that have not been suppressed. Hysteresis uses two thresholds and if the magnitude is below the first threshold, it is set to zero (made a non-edge). If the magnitude is above the high threshold, it is made an edge. And if the magnitude is between the two thresholds, then it is set to zero.

Applying the above methods, the detection results are shown in Fig.2. Fig.2 (a) shows the original image. Fig.2 (b) shows the edge detection with Sobel operator. Fig.2 (c) shows the edge detection with Laplace operator. Fig.2 (d) shows the edge detection with Canny operator.

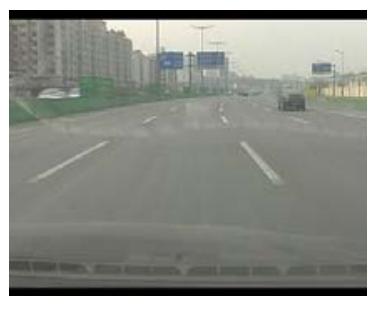

(a)

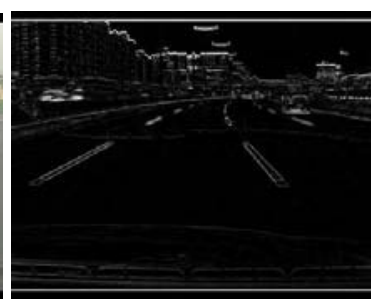

(b)

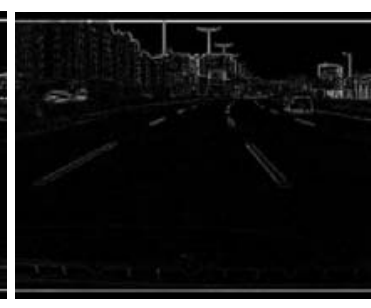

(c)

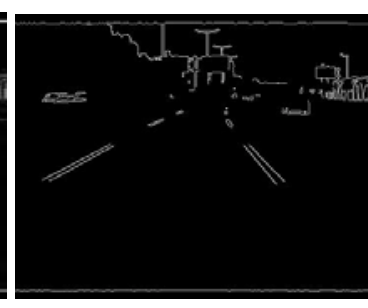

(d)

Figure 2. Geometric model of system imaging

Obviously, the Canny edge detection algorithm is the most effective method for edge detection. Therefore, we use the Canny detector in this subject.

\section{B. Linear Model Fitting}

Lanes can be treated as the straight lines. Hough transform is an efficient way to detect the straight lines. Hough transform achieve a kind of mapping from the image space to parameter space. It has very good fault tolerance and robustness. The basic principle of Hough transform is to use duality between point and line to change the curve given by the original image space into a point of the parameter space through the curve expression. Therefore, the problem of detection given curve in the original image is transformed into the problem of finding the peak in the parameter space. In other word, that is to transform detecting the overall characteristics into detecting the local characteristics, such as beeline, oval, round, arc and so on.

The output Hough transform is a set of straight lines, some of which may be the lane marking lines. Because highway lane marking lines are structured, a set of requirements is identified to exclude certain lines from the candidate lane marking lines. By controlling the angle and length of lines, and 
the minimum distance between segments on the same straight line, the ideal lane has been extracted successfully. The difference is shown in Fig.5, Fig.5 (a) shows the result of inappropriate setting Hough transform, and Fig.5 (b) shows the result of appropriate setting.

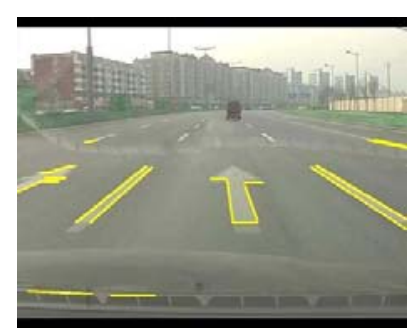

(a)

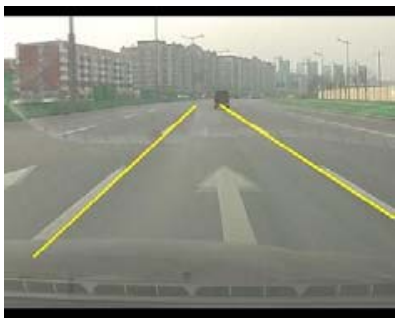

(b)

Figure 3. $\quad$ Lines extracted after Hough transform

\section{Setting of the Region of Interest (ROI)}

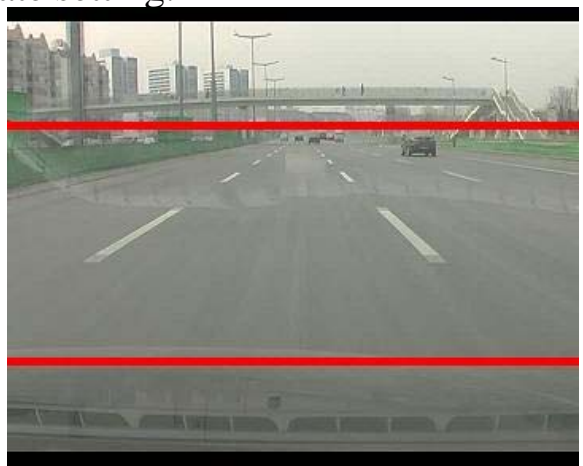

Figure 4. $\quad$ Lines extracted after Hough transform

The amount of computation of real-time lane tracking processing is very large, however, due to the need of certain road image information, we just need to analyze and find expected characteristics only in the Region of Interest, instead of analyzing the entire image. Setting the Region of Interest can significantly accelerate the speed of the lane detection, reduce hardware requirements, and meet the real-time requirement. In addition, it can also reduce the interference of useless information to reduce the error rate greatly. Of course, how to choose the Region of Interest is critical, because choose the wrong Region of Interest will result in the failure detection.

Fig.6 shows a typical lane picture. The lane information that we need only exists in the middle region, while the other regions just contain useless information. The lane information that we need only exists in the middle region, while the other regions just contain useless information.

\section{Experiments}

Fig. 5 shows experimental facilities installed in the test vehicle. And to validate the performance of this lane detection method, a driver drove the test vehicle on different sections of expressway in different light conditions

Fig.6 shows the detection result of clear day noon (a), cloudy day (b), nightfall (c), and lane with only one side normal marking (d).

\section{Conclusions}

In this paper, a lane detection method for Lane Departure Warning System is proposed. Experiment applying several edge detection operators shows that the Canny detector is the most suitable method for edge detection. Hough transform with appropriate setting is selected as the efficient way to detect straight lines. Setting the Region of Interest accelerates the speed of the lane detection significantly and reduces the error rate greatly. At last, experiment results indicate that this lane detection method can extract lane information from road images acquired efficiently and accurately. 


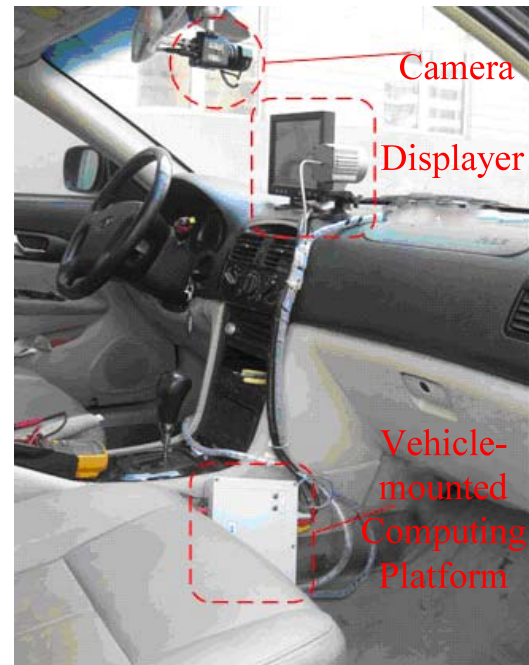

Figure 5. Experimental Facilities

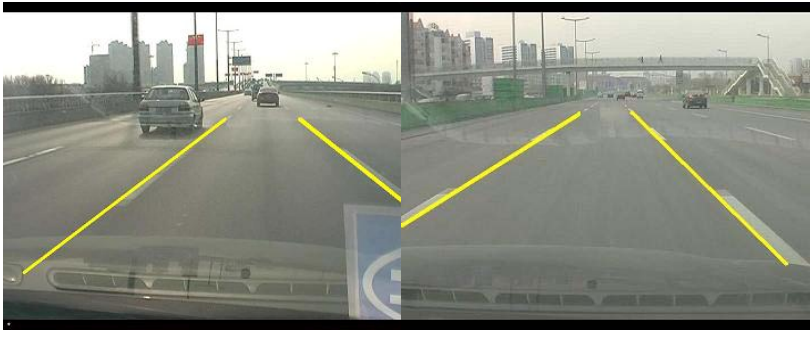

(a)

(b)

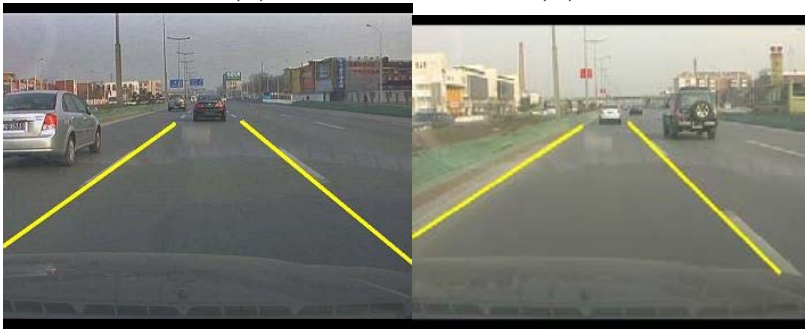

(c)

(d)

Figure 6. Detection Result in Different Conditions

\section{References}

[1] Xiangjing An, Mo Wu, Hangen He, "A Novel Approach to Provide Lane Departure Warning Using Only One Forward-Looking Camera," Proc. International Symp. Collaborative Technologies and Systems, 2006 (CTS 2006), May 2006 ,pp.356-362, doi:10.1109/CTS.2006.9.

[2] Younguk Yim, Se-Young Oh, "Three-feature based automatic lane detection algorithm (TFALDA) for autonomous driving," Proc. IEEE/IEEJ/JSAI International Conference on Intelligent Transportation Systems, 1999 (ITS1999), IEEE Press, pp.929 - 932, doi:10.1109/ITSC.1999.821188.

[3] W.Kwon,J.-W.Lee,D.Shin, "Experiments on decision making strategies for a lane departure warning system,” Proc. IEEE International Conference on Robotics and Automation, 1999, IEEE Press, 1999, pp. 2596 - 2601 vol.4, doi:10.1109/ROBOT. 1999.773988.

[4] H. Godthelp, P. Milgram and G. Blaauw, "The development of a time related measure to describe driving strategy”. Human Factors, vol. 26, no. 3, pp. 257-268, 1984.

[5] Jung, C.R. Kelber, C.R, “A lane departure warning system using lateral offset with uncalibrated camera ,” Proc. IEEE Intelligent Transportation Systems 2005, IEEE Press, Sept. 2005, pp. 102-107, doi:10.1109/ITSC.2005.1520073.

[6] Risack, R. Mohler, N. Enkelmann, W. Fraunhofer, “A video-based lane keeping assistant," Proc. IEEE Intelligent Vehicles Symposium, IV 2000, IEEE Press, 2000, pp. 356-361, doi:10.1109/IVS.2000.898369.

[7] Rafael C. Gonzalez, Richard E. Woods, Digital image processing, 2nd ed., New Jersey: Prentice-Hall,2002. 